\title{
Chapter 15 \\ Testing Fundamental Physics by Using Levitated Mechanical Systems
}

\author{
Hendrik Ulbricht
}

\begin{abstract}
We will describe recent progress of experiments towards realising largemass single particle experiments to test fundamental physics theories such as quantum mechanics and gravity, but also specific candidates of Dark Matter and Dark Energy. We will highlight the connection to the work started by Otto Stern as levitated mechanics experiments are about controlling the centre of mass motion of massive particles and using the same to investigate physical effects. This chapter originated from the foundations of physics session of the Otto Stern Fest at Frankfurt am Main in 2019, so we will also share a view on the Stern Gerlach experiment and how it related to tests of the principle of quantum superposition.
\end{abstract}

\section{Introductory Remarks}

Experimentally, this research programme is about gas-phase experiments with largemass particles, large compared to the mass of a single hydrogen atom, in order to test fundamental theories without the influence of the environment, which typically results in coherence-spoiling noise and decoherence effect. Tests of fundamental theories, such as quantum mechanics and gravity, are in the low-energy regime of non-relativistic velocities and therefore far away from a parameter regime of highenergy particle physics considerations. Fundamental theories will be tested in a new regime.

While Otto Stern's pioneering experiments [1], aligned with a fantastically bold and clear research programme, were about the study and control of freely propagating atoms and molecules in particle beams, we here make use of optical, magnetic and electric fields to trap and manipulate single particles, consisting of many atoms, in order to study the new physics and chemistry. The challenge here is to have a strong enough handle on the motion of the particle. For instance, the optical dipole force $F=\alpha \nabla E^{2}$ is strong and able to trap individual atoms and atomic ensembles making use of resonance effects. This is impossible for large molecules, again such

H. Ulbricht $(\varangle)$

School of Physics and Astronomy, University of Southampton, Highfield SO17 1BJ, UK

e-mail: h.ulbricht@soton.ac.uk

B. Friedrich and H. Schmidt-Böcking (eds.), Molecular Beams in Physics and Chemistry, https://doi.org/10.1007/978-3-030-63963-1_15 
which consist of many atoms, as resonances are manifold and the oscillator strength is distributed across many different state transitions far away from the ideal two-level system situation which we luckily find in some atoms, which gave rise to a revolution in experimental physics. Cold atom experiments now allow for ultra-precise control of various degrees of freedoms - including the centre of mass motion of the atoms and to prepare non-classical sates, including collective ones such as atomic Bose Einstein Condensates (BEC). In a way our programme aims to achieve a similar level of control, but for particles of large mass and different cooling and manipulation techniques have to be developed and used for that. The off-resonant dipole force where $\alpha$ is a measure of the off-resonant detuning of all affected molecular states is however too weak to lead to a large enough effect to trap and manipulate individual molecules by coherent laser light $[2,3]$. This situation changes dramatically if one increases the size (volume $V$ ) of the particle to trap and therefore its polarizability $\alpha \propto V$. Then dipole force becomes so strong to form a deep optical trap and optical fields can be used for controlling single particle motions again, which gave rise to the development of the new research field levitated optomechnaics [4], based on early pioneering work by Arthur Ashkin (Nobel Prize in Physics in 2018) [5] and already then in close relation to the then soon to be called cold atomic and optical physics. By now the field of levitated large-mass particle systems has seen the implementation of other than optical forces for trapping and manipulation, namely time-varying electrical fields in Paul traps [6] and magnetic traps [7], sometimes including superconductors [8]. All such technical developments give rise to the hope to soon perform experiments with truly macroscopic quantum systems, outperforming existing paradigms of large-mass matterwave interferometry [9]. Macroscopic here entails the involvement of a large-mass particle in a quantum superposition of large spatial separation [10].

There are two pillars of our research programme on testing fundamental physics are with a certain methodological approach. The first is the clearly distinctive predictions for the outcome of the same experiment originated from alternative theoretical descriptions. This is our approach for testing the universality of the quantum superposition principle in the context of collapse models [11]. Quantum mechanics and collapse models predict a different outcome of a matterwave interferometry experiment-if the experiment is performed in the right parameter regime. The second pillar of our research programme is to first perform a detailed analysis of the new physics to be tested and then to chose the best experiment to perform the test.

Outlook of this chapter. In the following, we will address new avenues to test quantum mechanics in Sect. 2 with the specific emphasis on experiments using levitated mechanical systems. Then we will address experimental tests of the interplay between quantum mechanics and gravity in Sect. 3 including the discussion of the semiclassical Schrödinger-Newton equation, gravitational deocherence of the wavefunction and the gravity of a quantum state. In the final Sect. 4 we will refer to using the Wigner function to simulate the original Stern Gerlach experiment. 


\section{Testing Quantum Mechanics with Collapse Models}

There is an increasing interest in developing experiments aimed at testing collapse models, in particular the Continuous Localization Model (CSL), the natural evolution of the GRW model initially proposed by Ghirardi et al. [11-14]. Current experiments and related bounds on collapse parameters are partially discussed in other contributions in this review. Our aim here is to discuss some of the most promising directions towards future improvements. We will mostly focus on non-interferometric experiments. In Sect.2.1 we will briefly outline proposals of matter-wave interference with massive nano/microparticles. Finally, in Sect. 2.2 we will discuss mechanical experiments, in particular ongoing experiments with ultracold cantilevers, ongoing and proposed experiments based on levitated nanoparticles and microparticles. We will not consider here two important classes of experiments which are separately discussed by other contributors in this review: matter-wave interference with molecules and space-based experiments. We will end in Sect. 2.3 with some ideas on how precision experiments can be used for testing collapse models. A summary of recent interferometric and non-interferomtric experiments which could set direct bounds on the CSL collapse model are summarized in Fig. 1.
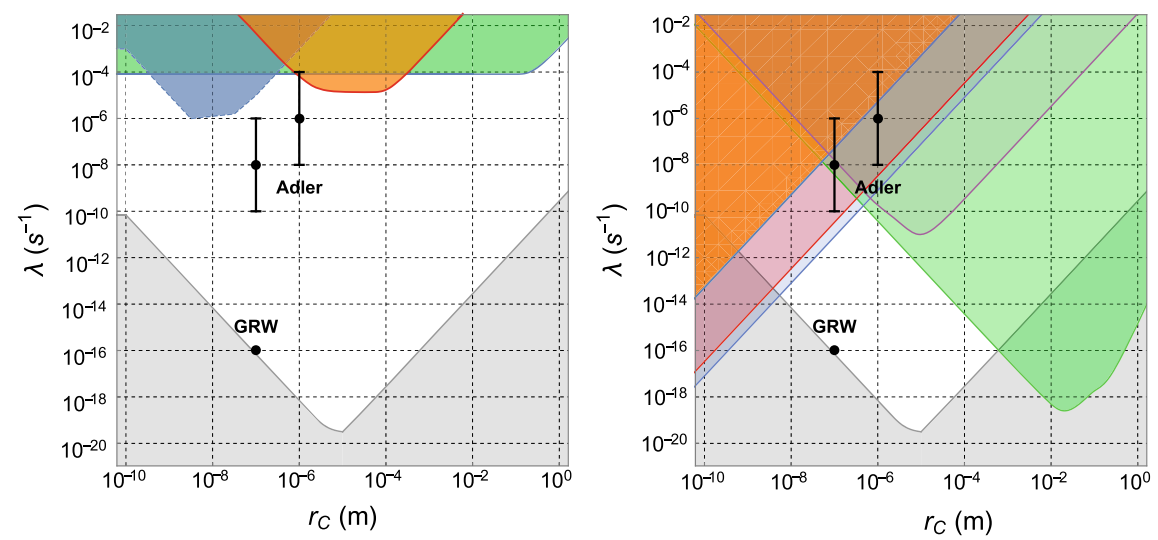

Fig. 1 Exclusion plots for the CSL parameters with respect to the GRW and Adler theoretically proposed values $[12,15]$. Left panel-Excluded regions from interferometric experiments: molecular interferometry [16] (blue area), atom interferometry [17] (green area) and experiment with entangled diamonds [18] (orange area). Right panel—Excluded regions from non-interferometric experiments: LISA Pathfinder [19, 20] (green area), cold atoms [21] (orange area), phonon excitations in crystals [22] (red area), X-ray measurements [23] (blue area) and nanomechanical cantilever [24]. We report with the grey area the region excluded based on theoretical arguments [25]. Figure and caption taken from [26] 


\subsection{Tests of Quantum Mechanics by Matter-Wave Interferometry}

Matterwave interferometry is directly testing the quantum superposition principle. Relevant for mass-scaling collapse models, such as CSL, are matterwave interferometers testing the maximal macroscopic extend in terms of mass, size and time of spatial superpositions of single large-mass particles. Such beautiful, but highly challenging experiments have been pushed by Markus Arndt's group in Vienna to impressive particle masses of $10^{4}$ atomic mass units (amu), which still not significantly challenging CSL. Therefore the motivation remains to push matterwave interferometers to more macroscopic systems. Predicted bounds on collapse models set by large-mass matterwave interferometers are worked out in detail in [27].

As usual in open quantum system dynamics treatments, non-linear stochastic extensions of the Schrödinger equation on the level of the wavefunction [28] correspond to a non-uniquely defined master equation on the level of the density matrix $\rho$ to describe the time evolution of the quantum system, say the spatial superposition across distance $|x-y|$, where the conserving von Neumann term $\partial \rho_{t}(x, y) / \partial t=-(i / \hbar)[H, \rho]$, is now extended by a Lindblad operator $L$ term:

$$
\frac{\partial \rho_{t}(x, y)}{\partial t}=-\frac{i}{\hbar}\left[H, \rho_{t}(x, y)\right]+L \rho_{t}(x, y),
$$

where $H$ is the Hamilton operator of the quantum system and different realisations of a Lindblad operator are used to describe both standard decoherence (triggered by the immediate environment of the quantum system) [29] as well as spontaneous collapse of the wavefunction triggered by the universal classical noise field as predicted by collapse models.

Now the dynamics of the system is very different with and without the Lindbladian, where with the Lindbladian the unitary evolution breaks down and the system dynamics undergoes a quantum-to-classical transition witnessed by a vanishing of the fringe visibility of the matterwave interferometer. In the state represented by the density matrix the off-diagonal terms vanish as the system evolves according to the open system dynamics, the coherence/superposition of that state is lost. The principal goal of interference experiments with massive particles is then to explore and quantify the relevance of the $\left(L \rho_{t}(x, y)\right)$-term-as collapse models predict a break down of the quantum superposition principle for a sufficient macroscopic system. An intrinsic problem is the competition with known and unknown environmental decoherence mechanisms, if a visibility loss is observed. However solutions seem possible.

In order to further increase the macroscopic limits in interference some ambitious proposals have been made utilizing nano- and micro-particles, c.f. Fig. 2. The main challenge is to allow for a long enough free evolution time of the prepared quantum superposition state in order to be sensitive to the collapsing effects. The free evolution - the spatial spreading of the wavefunction $\Psi(r, t)$ with time-according to the time-dependent Schrödinger equation with the potential $V(r)=0$, 


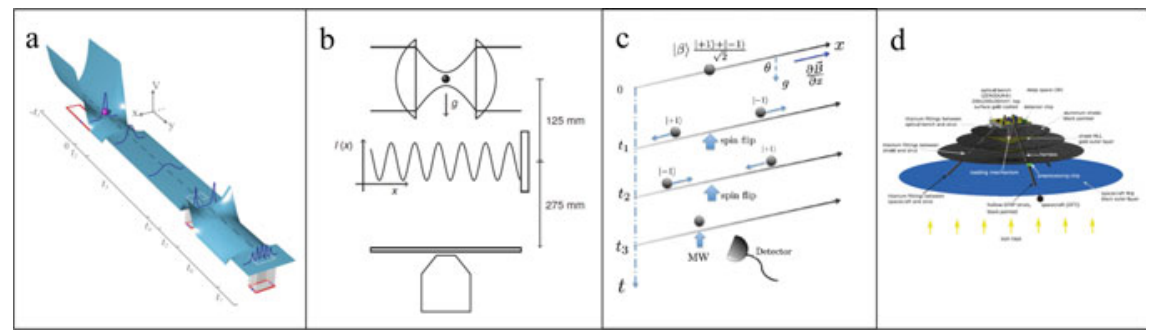

Fig. 2 Illustration of some of the proposed schemes for matterwave interferometry with nanoand micro-particles to test the quantum superposition principle directly, and therefore also collapse models. a The cryogenic skatepark for a single superconducting micro-particle (adapted from Ref. [31]); b The nanoparticle Talbot interferometer (adapted from Ref. [30]); $\mathbf{c}$ The Ramsey scheme addressing the electron Spin of a NV-centre diamond coupled to an external magnetic field gradient $(\partial B / \partial x)$ (adapted from Ref. [32]); $\mathbf{d}$ The adaptation of an interferometer at a free falling satellite platform in space to allow form longer free evolution times (adapted from Ref. [33])

$$
\frac{\partial}{\partial t} \Psi(r, t)=-i \frac{\hbar}{2 m} \nabla^{2} \Psi(r, t)
$$

describes a diffusive process for probability amplitudes similar to a typical diffusion equation with the imaginary diffusion coefficient $(-i \hbar / 2 m)$. Therefore the spreading of $\Psi(r, t)$ scales inverse with particle mass $m$. For instance for a $10^{7}$ amu particle it already takes so long to show the interference pattern in a matterwave experiment that the particle would significantly drop in Earth's gravitational field, in fact it would drop on the order of $100 \mathrm{~m}$. This requires a dramatic change in the way large-mass matterwave interferometry experiments have to be performed beyond the mass of $10^{6}$ amu [30].

Different solutions are thinkable. One could of course envisage building $100 \mathrm{~m}$ fountain, but that seems very unfeasible also given that no sufficient particle beam preparation techniques exist (and don't seem to be likely to be developed in the foreseeable future) to enable the launch and detection of particles in the mass range in question over a distance of $100 \mathrm{~m}$. One can consider to levitate the particle by a force field to compensate for the drop in gravity, but here we face a high demand on the fluctuations of that levitating field, which have to be small compared to the amplitudes of the quantum evolution, which is not feasible with current technology. A maybe possible option os to coherently boost/accelerate the evolution of the wavefunction spread by a beam-splitter operation. The proposals in Refs. [31, 32] are such solutions, which are still awaiting their technical realisation for large masses. Alternatively and more realistic given technical capability is to allow for long enough free evolution by freely fall the whole interferometer apparatus in a co-moving reference frame with the particle. This is the idea of the MAQRO proposal, a dedicated satellite mission in space to perform large-mass matterwave interference experiments with micro- and nano-particles [33]. 
Another interesting approach is to consider the use of cold or ultra-cold ensembles of atoms such as cloud in a magneto optical trap (MOT) or an atomic Bose-Einstein Condensate (BEC) as also there we find up to $10^{8}$ atoms of alkali species such as rubidium or caesium. On closer look it turns out that such weakly interacting atomic ensembles are not of immediate use for the purpose to test macroscopic quantum superpositions in the context of collapse model test. For instance testing the CSL model is build on a mass (number of particles $N$, more precisely the number of nucleons: protons and neutrons in the nuclei of the atoms) amplification which in principle can even go with $N^{2}$, if the condition for coherent scattering of the classical collapse noise treated as a wave with correlation length $r_{c}$ scattered at the particle in the quantum superposition state. The central assumption of this amplification mechanism is that if the CSL noise is collapsing the wavefunction of only one of the constituent nucleons, then the total wavefunction of the whole composite object collapses. While in the case of a nanoparticle consisting of many atoms (and therefore nucleons), it is not the case for an weakly interacting atomic ensemble. If one atom is collapsing then the total atomic wavefunction remains intact and the one atom is lost from the ensemble.

This may change if the atoms in the cold or ultra-cold ensemble can be made stronger interacting, without running into the complications of chemistry which may forbid condensation of the atomic - then molecular-cloud at all. However there is hope that quantum optical state preparation techniques applied after a BEC has been formed such as collective NOON or squeezed states enable $N$ and even $N^{2}$ scaling in the fashion fit for testing wavefunction collapse.

Interestingly, this might be different if the physical mechanism responsible for the collapse of the wavefunction, which remains highly speculative at present, is in any way related to gravity [34], then there might be hope that atomic ensembles even in the weakly interacting case can be used to test CSL-type models. The condition to fulfil is that the atomic ensemble is interacting gravitationally strong enough so that it acts collectively under collapse, even if just a single constituent atom (nucleon) is affected by the collapsing effect.

\subsection{Non-interferometric Mechanical Tests of Quantum Mechanics}

This class of experiments has emerged in recent years as one of the most powerful and effective ways to test collapse models. The underlying idea [35, 36] is that a mechanism which continuously localizes the wavefunction of a mechanical system, which can be either a free mass or a mechanical resonator, must be accompanied by a random force noise acting on its center-of-mass. This leads in turn to a random diffusion which can be possibly detected by ultrasensitive mechanical experiments.

In a real mechanical system such diffusion will be masked by standard thermal diffusion arising from the coupling to the environment, i.e. from the same effects 
which lead to decoherence in quantum interference experiments [37]. In practice there will be additional non-thermal effects, due to external non-equilibrium vibrational noise (seismic/acoustic/gravity gradient). Moreover, one has to ensure that the backaction from the measuring device is negligible.

Under the assumption that thermal noise is the only significant effect, the (onesided) power spectral density of the force noise acting on the mechanical system is given by:

$$
S_{f f}=\frac{4 k_{B} T m \omega}{Q}+2 \hbar^{2} \eta .
$$

where $k_{B}$ is the Boltzmann constant, $T$ is the temperature, $m$ is the mass, $\omega$ the angular frequency, $Q$ is the mechanical quality factor.

$\eta$ is a diffusion constant associated to spontaneous localization, and can be calculated explicitly for the most known models. For CSL, it is given by the following expression

$$
\begin{aligned}
\eta & =\frac{2 \lambda}{m_{0}^{2}} \iint \mathrm{d}^{3} \mathbf{r} \mathrm{d}^{3} \mathbf{r}^{\prime} \exp \left(-\frac{\left|\mathbf{r}-\mathbf{r}^{\prime}\right|^{2}}{4 r_{C}^{2}}\right) \frac{\partial \varrho(\mathbf{r})}{\partial z} \frac{\partial \varrho\left(\mathbf{r}^{\prime}\right)}{\partial z^{\prime}} \\
& =\frac{(4 \pi)^{\frac{3}{2}} \lambda r_{C}^{3}}{m_{0}^{2}} \int \frac{\mathrm{d}^{3} \mathbf{k}}{(2 \pi)^{3}} k_{z}^{2} e^{-\mathbf{k}^{2} r_{C}^{2}}|\tilde{\varrho}(\mathbf{k})|^{2}
\end{aligned}
$$

with $\mathbf{k}=\left(k_{x}, k_{y}, k_{z}\right), \tilde{\varrho}(\mathbf{k})=\int \mathrm{d}^{3} \mathbf{x} e^{i \mathbf{k} \cdot \mathbf{r}} \varrho(\mathbf{r})$ and $\varrho(\mathbf{r})$ the mass density distribution of the system. In the expressions above $m_{0}$ is the nucleon mass and $r_{C}$ and $\lambda$ are the free parameters of CSL. The typical values proposed in CSL literature are $r_{C}=10^{-7}$ $\mathrm{m}$ and $10^{-6} \mathrm{~m}$, while for $\lambda$ a wide range of possible values has been proposed, which spans from the GRW value $\lambda \approx 10^{-16} \mathrm{~Hz}[12,13]$ to the Adler value $\lambda \approx 10^{-8 \pm 2}$ $\mathrm{Hz}$ at $r_{C}=10^{-7} \mathrm{~m}$ [15]. The possibility for such non-interferometric tests, which aim to directly test the non-thermal noise predicted by collapse models [24, 38-41].

An experiment looking for CSL-induced noise has to be designed in order to maximize the 'noise to noise' ratio between the CSL term and the thermal noise. In practice this means lowest possible temperature $T$, lowest possible damping time, or linewidth, $1 / \tau=\omega / Q$, and highest possible $\eta / m$ ratio. The first two conditions express the requirement of lowest possible power exchange with the thermal bath, the third condition is inherently related to the details of the specific model.

For CSL we can distinguish two relevant limits. When the characteristic size $L$ of the system is small, $L \ll r_{C}$, then the CSL field cannot resolve the internal structure of the system, and one finds $\eta / m \propto m$. When the characteristic length of the system in the direction of motion $L$ is large, $L \gg r_{C}$, then $\eta / m \propto \rho / L$, where $\rho$ is the mass density $[24,38,40]$. The expressions in the two limits imply that, for a well defined characteristic length $r_{C}$, the optimal system is a plate or disk with thickness $L \sim r_{C}$ and the largest possible density $\rho$.

Among other models proposed in literature, we mention the gravitational DiosiPenrose (DP) model, which leads to localization and diffusion similarly to CSL. The diffusion constant $\eta_{D P}$ is given by [40]: 


$$
\eta_{D P}=\frac{G \rho m}{6 \sqrt{\pi} \hbar}\left(\frac{a}{r_{D P}}\right)^{3},
$$

where $a$ is the lattice constant and $G$ is the gravitational constant, so that he ratio $\eta_{D P} / m$ depends only on the mass density. Unlike CSL, there is no explicit dependence on the shape or size of the mechanical system.

\subsubsection{Levitated Mechanical Systems}

One of the most promising approaches towards a significant leap forward in the achievable sensitivity to spontaneous collapse effects is by levitation of nanoparticles or microparticles. The main benefits of levitation are the absence of clamping mechanical losses and wider tunability of mechanical parameters. In addition, several degrees of freedom can be exploited, either translational or rotational [41, 42]. This comes at the price of higher complexity, poor dynamic range and large nonlinearities, which usually require active feedback stabilization over multiple degrees of freedom. However, levitated systems hold the promise of much better isolation from the environment, therefore higher quality factor. One relevant example, in the macroscopic domain, is the space mission LISA Pathfinder, which is based on an electrostatically levitated test mass, currently setting the strongest bound on collapse models over a wide parameter range [43].

Several levitation methods for micro/nanoparticles are currently being investigated. The most developed is optical levitation using force gradients induced by laser fields, the so called optical tweezer approach [5]. While this is a very effective and flexible approach to trap nanoparticles, in this context it is inherently limited by two factors: the relatively high trap frequency, in the order of $100 \mathrm{kHz}$, and the high internal temperature of the particles, induced by laser power absorption, which leads ultimately to strong thermal decoherence. Alternative approaches have to be found, featuring lower trap frequency and low or possible null power dissipated in the levitated particle. The two possible classes of techniques are electrical levitation and magnetic levitation.

Electrical levitation has been deeply developed in the context of ion traps. The standard tool is the Paul trap, which allows to trap an ion, or equivalently a charged nanoparticle, using a combination of ac and dc bias electric fields applied through a set of electrodes [44]. The power dissipation is much lower than in the optical case, and the technology is relatively well-established. However, the detection of a nanoparticle in a Paul trap still poses some technological challenge (Fig. 3).

This issue has been extensively investigated in a recent paper [45], specifically considering a nanoparticle in a cryogenic Paul trap in the context of collapse model testing. Three detection schemes have been considered: an optical cavity, an optical tweezer, and a all-electric readout based on SQUID. It was found that to detect the nanoparticle motion with good sensitivity, optical detection has to be employed. Unfortunately, optical detection is not easily integrated in a cryogenic environment, and leads to a nonnegligible internal heating and excess force noise. On the other 


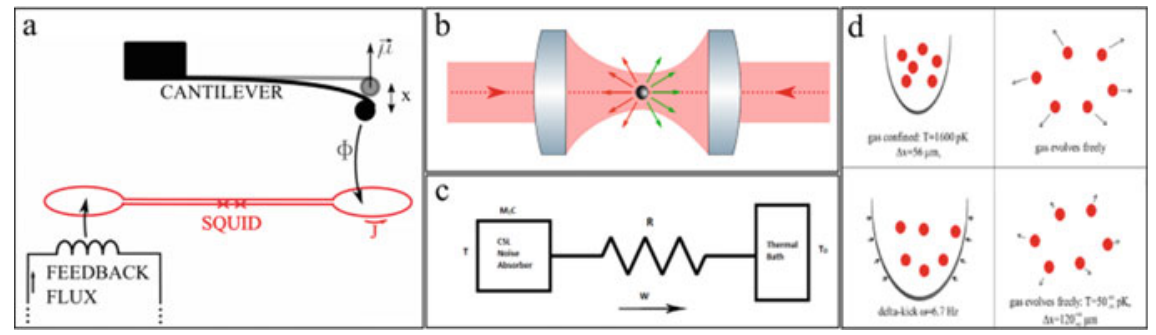

Fig. 3 Simplified sketch of some of the noninterferometric methods to test collapse models discussed in this contribution. a Measuring the mechanical noise induced by CSL using an ultracold cantilever detected by a SQUID (adapted from Ref. [24]); $\mathbf{b}$ Measuring the mechanical noise induced by CSL using a levitated nanoparticle detected optically (adapted from Ref. [45]); $\mathbf{c}$ Measuring the heating induced by CSL in a solid matter object cooled to very low temperature (adapted from Ref. [46]); $\mathbf{d}$ Measuring the increase of kinetic energy induced by CSL in a ultracold atoms cloud (adapted from Ref. [47])

hand, an all-electrical readout would potentially allow for a better ultimate test of collapse models, but at the price of a very poor detection sensitivity, which could make the experiment hardly feasible. The authors argue that a Paul-trapped nanoparticle, with an oscillating frequency of $1 \mathrm{kHz}$, cooled in a cryostat at $300 \mathrm{mK}$ with an optical readout may be able to probe the CSL collapse rate down to $10^{-12} \mathrm{~Hz}$ at $r_{C}=10^{-7}$ m. A SQUID-based readout, if viable, could theoretically allow to reach $10^{-14} \mathrm{~Hz}$.

A recent experiment employing a nanoparticle in a Paul trap with very low secular frequencies at $\sim 100 \mathrm{~Hz}$ and low pressure has demonstrated ultranarrow linewidth $\gamma / 2 \pi=82 \mu \mathrm{Hz}$ [48]. This result has been used to set new bounds on the dissipative extension of CSL. This experiment may be able to probe the current limits on the CSL model in the near future, once it will be performed at cryogenic temperature and the main sources of excess noise, in particular bias voltage noise, will be removed.

Magnetic levitation, while less developed, has the crucial advantage of being completely passive. Furthermore the trap frequencies can be quite low, in the $\mathrm{Hz}$ range. Three possible schemes can be devised: levitation of a diamagnetic insulating nanoparticle with strong external field gradients [49, 50], levitation of a superconducting particle using external currents [8,51-53], and levitation of a ferromagnetic particle above a superconductor [54].

The first approach has been recently considered in the context of collapse models [50]. The experiment was based on a polyethylene glycol microparticle levitated in the static field generated by neodymium magnets and optical detection. The experiment has been able to set an upper bound on the CSL collapse rate $\lambda<10^{-6.2} \mathrm{~Hz}$ at $r_{C}=10^{-7} \mathrm{~m}$, despite being performed at room temperature. A cryogenic version of this experiment should be able to approach the current experimental limits on CSL.

The second and third approach based on levitating superconducting particles are currently investigated by a handful of groups [8,51-54], but no experiment has so far reached the experimental requirements needed to probe collapse models. However, a significant progress has been recently achieved: a ferromagnetic microparticle 
levitated above a type I superconductor (lead) and detected using a SQUID, has demonstrated mechanical quality factors for the rotational and translational rigid body mechanical modes exceeding $10^{7}$, corresponding to a ringdown time larger than $10^{4}$ seconds [53]. The noise is this experiment is still dominated by external vibrations. However, as the levitation is completely passive and therefore compatible with cryogenic temperatures, this appears as an excellent candidate towards near future improved tests of collapse models.

\subsection{Concluding Remarks on Testing Quantum Mechanics in the Context of Collapse Models}

We have discussed avenues for non-interferometric and interferometric tests of the linear superposition principle of quantum mechanics in direct comparison to predictions from collapse models which break the linear/unitary evolution of the wavefunction. As matters stand both non-interferometric and interferometric set already bounds on the CSL collapse model, while those from non-interferometric tests are stronger by orders of magnitude. The simple reason lyes in the immense difficulty to experimentally generate macroscopic superposition states, however a number of proposals have been made and experimentalists are set to approach the challenge.

We want to close by mentioning that there are possible other experimental platforms which could set experimental bunds on collapse models and it would be of interest to study those in detail. Collapse models predict a universal classical noise field to fill the Universe and in principle couple to any physical system. In the simplest approach the experimental test particle can be regarded as a two-level system, as typically described in quantum optics. Then the collapse noise perturbs the two-level system and emissive broadening and spectral shifts can be expected, unfortunately out of experimental reach at the moment [55]. The minuscule collapse effect on a single particle (nucleon) needs some sort of amplification mechanism which usually comes with an increase of the number of constituent particles. However, ultra-high precision experiments have improved a lot in recent years. For instance much improved ultrastable Penning ion traps are used to measure the mass of single nuclear particles, such as the electron, proton, and neutron, with an ultra-high precision to test quantum electrodynamics predictions [56]. In principle also here the effect of collapse models should become apparent. Any theoretical predictions are difficult as relativistic versions of collapse models still represent a serious formal challenge. Other high potentials for testing collapse are ever more precise spectroscopies of simple atomic species with analytic solutions such as transitions in hydrogen [57] and needless to say atomic clocks [58].

As tests move on to set stronger and stronger bounds, we have to remain open to actually find something new. It is so easy to disregard tiny observed effects as unknown technical noise. In the case of direct testing collapse noise it is a formidable theoretical challenge to think about possible physics responsible for collapse, satisfy- 
ing the constrains given by the structure of the collapse equation: the noise has to be classical and stochastic. Such concrete physics models will predict a clear frequency fingerprint, should we ever observe the collapse noise field.

\section{Testing the Interplay Between Quantum Mechanics and Gravity}

Here we will be concerned with table-top experiments in the non-relativistic regime as these experiments may provide a new access to shine light on the quantumgravity interplay. Therefore the main emphasis is to explore possible routes to enter the new parameter regime, where both quantum mechanics and gravity are significant, see Fig. 4). This means the mass of the object has to be large enough to show gravity effects while also not being too large to still allow for the preparation of non-classical features of the behaviour of that massive object. That regime where both physical effects, the quantum and the gravity, could be expected to be relevant is at around the Planck mass, which is derived from the right mixture of fundamental constants ( $\hbar$ Planck's constant, $c$ speed of light, $G$ gravitational constant) $m_{p l}=\sqrt{\hbar c / G}=2.176470(51) \times 10^{-8} \mathrm{~kg}$ (the official CODATA, NIST) or below. No quantum experiment has been performed in that mass range.

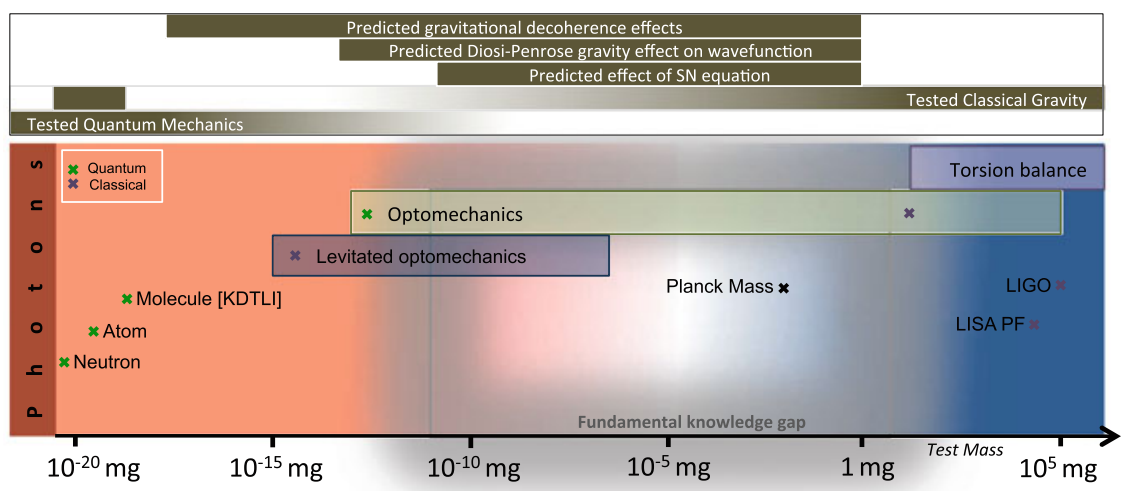

Fig. 4 Exploration map of mass: Mass range of the test mass as explored by experiments. Experiments to detect gravity have been done in the classical domain, right hand side of picture, with comparable large masses. Quantum experiments are routinely performed by using objects of much smaller masses so that gravity effects do not become visible or relevant. Neutron and atom matterwave interferometers are different as the test mass there is very small [the mass of a single neutron or atom], but in a spatial superposition state. The desired mass range for-at least some of - the experiments summarized in this review article is at the overlap between sufficiently large mass to see significant effects of gravity of the particle itself, while the particle can be maintained in a non-classical state. The domain where massive particles can be prepared in such non-classical states is on the left hand side of the picture 
When we refer to quantum mechanical behaviour of massive systems, we mean the centre of mass motion of such a system, which may consist of many atoms. Surely, there are many other [we call those internal] degrees of freedom of the same system such as electronic states or vibrations and rotations which are described as relative motions of the atoms forming the large object, but here we are not concerned with those. When we talk about superpositions, we mean spatial superpositions, in the sense of a the centre of mass of a single particle, which can be elementary or composite, being here and there at a given time, the Schrödinger cat state. The most massive complex quantum system, which has been experimentally put in such a superposition state, are complex organic molecules of a mass on the order of $m_{\max }=10^{-22} \mathrm{~kg}$ [9].

Typically for gravity experiments there are two masses involved, the source mass which generates a gravitational field, potential or curvature of space-time (the source mass has usually a big mass) and the test mass which is probing the gravity effect generated by the source mass. Torsion balances are the classic device for typical gravity experiments. We think there are two regimes interesting for experimental investigation: (1) the regime where a quantum system is the test mass and interacts with a large external source mass. This is the regime where neutron and atom interferometry are already very successful and provide tools for precise measurements of gravity effects. (2) the regime where the quantum system itself carries sufficient mass to be the source mass and to allow for related quantum gravity effects to become experimentally accessible. So far there has been no convincing experiment in the second regime. Any experiment performed in that second regime will ultimately give insight into the interplay between gravity and quantum mechanics. Test of the Schrödinger-Newton equation and of quantum effects in gravity fall in the latter regime. It may very well be that there are surprises waiting for us if we become able to probe that regime by experiments.

In the following we shall discuss the prospects to experimentally test the semiclassical Schrödinger-Newton equation, which plays also a role for some ideas of gravity induced collapse of the wavefunction such as put forward by Roger Penrose [59], gravitational decoherence such as some ideas to investigate the gravity effects within a spatial quantum superposition state.

\subsection{Proposals for Experimental Tests of the Schrödinger-Newton equation}

What is the gravitational field of a quantum system in a spatial superposition state? The seemingly most obvious approach, the perturbative quantization of the gravitational field in analogy to electromagnetism, makes it alluring to reply that the spacetime of such a state must also be in a superposition. The non-renormalizability of said theory, however, has also inspired the hypothesis that a quantization of the gravitational field might not be necessary after all $[60,61]$. Rosenfeld already expressed the 
thought that the question whether or not the gravitational field must be quantized can only be answered by experiment: There is no denying that, considering the universality of the quantum of action, it is very tempting to regard any classical theory as a limiting case to some quantal theory. In the absence of empirical evidence, however, this temptation should be resisted. The case for quantizing gravitation, in particular, far from being straightforward, appears very dubious on closer examination. [60]

Adopting this point of view, an alternative approach to couple quantum matter to a classical space-time is provided by a fundamentally semi-classical theory that is by replacing the source term in Einstein's field equations for the curvature of classical space-time, energy-momentum, by the expectation value of the corresponding quantum operator $[60,62]$ :

$$
R_{\mu \nu}+\frac{1}{2} g_{\mu \nu} R=\frac{8 \pi G}{c^{4}}\left\langle\Psi\left|\hat{T}_{\mu \nu}\right| \Psi\right\rangle .
$$

Of course, such presumption is not without complications. For instance, in conjunction with a no-collapse interpretation of quantum mechanics it would be in blatant contradiction to everyday experience [63]. Moreover, the nonlinearity that the backreaction of quantum matter with classical space-time unavoidably induces cannot straightforwardly be reconciled with quantum nonlocality in a causality preserving manner $[64,65]$. Be that as it may, there is no consensus about the conclusiveness of these arguments [66-68]. The enduring quest for a theory uniting the principles of quantum mechanics and general relativity gives desirability to having access to hypotheses which could be put to an experimental test in the near future.

In the non-relativistic limit, the assumption of fundamentally semi-classical gravity yields a non-linear, nonlocal modification of the Schrödinger equation, commonly referred to as the Schrödinger-Newton equation [34, 69, 70]. After a suitable approximation [70], for the center of mass of a complex quantum system of mass $M$ in an external potential $V_{\text {ext }}$ it reads:

$$
\begin{aligned}
\mathrm{i} \hbar \frac{\partial}{\partial t} \psi(t, \mathbf{r}) & =\left(\frac{\hbar^{2}}{2 M} \nabla^{2}+V_{\mathrm{ext}}+V_{g}[\psi]\right) \psi(t, \mathbf{r}) \\
V_{g}[\psi](t, \mathbf{r}) & =-G \int \mathrm{d}^{3} r^{\prime}\left|\psi\left(t, \mathbf{r}^{\prime}\right)\right|^{2} I_{\rho_{c}}\left(\mathbf{r}-\mathbf{r}^{\prime}\right)
\end{aligned}
$$

The self-gravitational potential $V_{g}$ depends on the wavefunction, and hence renders the equation nonlinear. The function $I_{\rho_{c}}$, which models the mass distribution of the considered system, will be defined below.

The Schrödinger-Newton equation has primarily been discussed in the context of gravitationally induced quantum state reduction [71, 72]. Its relevance for a possible experimental test of the necessity to quantize the gravitational field was pointed out by Carlip [61]. First ideas how to test such kind of nonlinear, self-gravitational effects focused on the spreading of a free wavefunction in matter-wave interferometry experiments [9, 37, 61, 69, 70]. Recently, other experimental test have been proposed 
including one based on the internal dynamics of a squeezed coherent ground state of a micron-sized silicon particle in a harmonic potential. We will now discuss further ideas for testing the Schrödinger-Newton equation.

\subsubsection{Proposed Direct Tests of Schrödinger-Newton Equation: Wavefunction Expansion}

The direct test of the Schrödinger-Newton (SN) equation is by studying the free expansion of the wavefunction of sufficiently massive objects. Then a contraction of the wave function according to the SN self-gravity effect should have a consequence on that expansion, competing with its natural Schrödinger's dynamics spread. Clearly, because to the weakness of gravitation interaction, the mass has to be sufficiently large while the object has to remain in a state which can be described by a centre of mass quantum wavefunction, meaning the spatial extent of the wavefunction should be detectable for the full duration of the evolution. See Fig. 5 for the mass-time parameter space required to observe the predicted SN effect directly, which has been studied extensively. While analytic solutions of the SN equation are difficult and even numerical simulations are non-trivial.

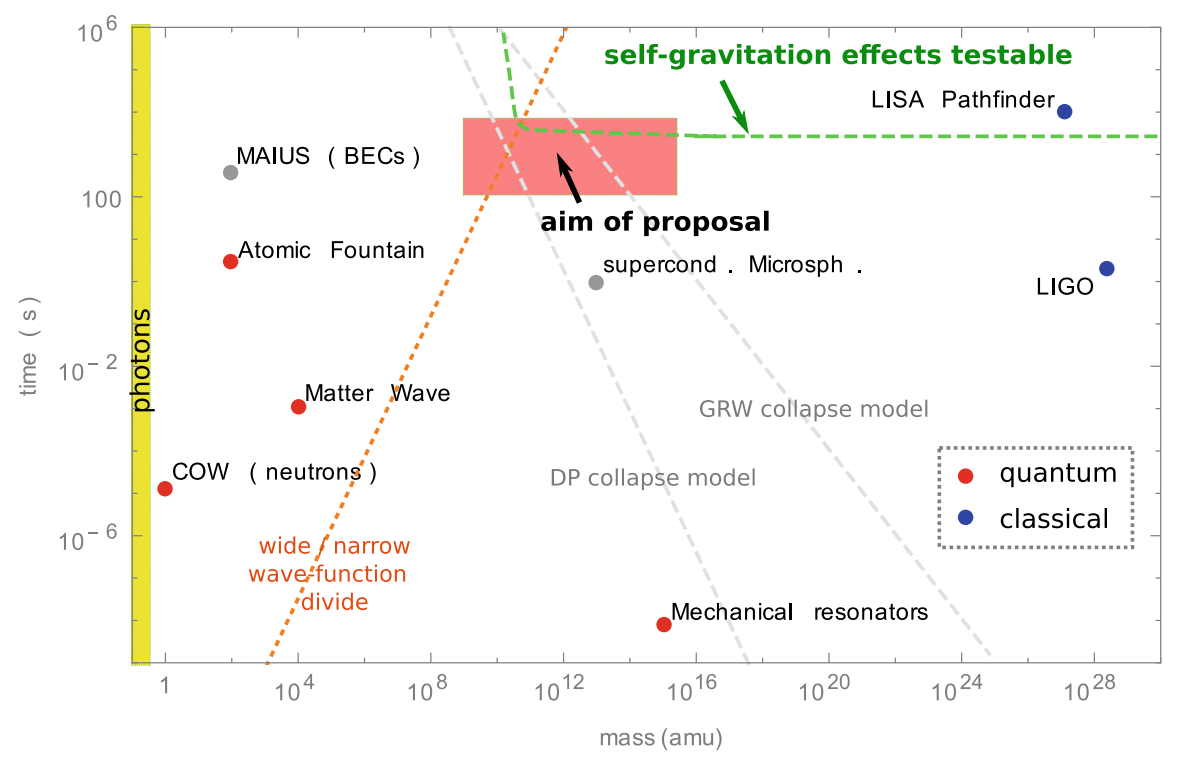

Fig. 5 Direct Test of Schrödinger-Newton (SN) wavefunction evolution: The mass-time plot to illustrate the parameter range which needs to be reached for direct SN wavefunction evolution experiments. This clearly needs to be done without external gravity and other forces/interactions and therefore an experiment in space appears a likely option. The red area shows the parameter range for a proposed space mission to test the $\mathrm{SN}$ effect 
One possible experimental scenario would be a molecule interferometry experiment [9]. While such matterwave experiments probe spatial superposition states of large molecules - the SN contraction effect could also be observed for a free expansion of a singular wave function originated from a point in space. The key is that the mass of the evolving quantum object has to be comparable large, much larger than the mass achieved in present molecule interferometry experiments. Cold atoms and even BEC of atoms, which benefit from the multitude of coherent manipulation, control and cooling schemes do not seem to have large enough mass in order to show the $\mathrm{SN}$ expansion/contraction effect. Clearly one needs a high mass at the same time as access to the coherent quantum evolution of the objects wavefunction. The high mass and the long expansion times to be studied challenge the experimental realisation.

Therefore, should direct tests of the SN equation be done in space? Yes, at this point there seems to be no other way to allow the wavefunction expansion for long enough, typically some hundred seconds, see Fig. 5. Proposals to levitate massive particles (optically or magnetically) and therefore to compensate for the drop in Earth's gravity have not been realised and are more problematic for SN test. The levitated tests rely on proposed techniques to accelerate the wavefunction expansion artificially by optical or magnetic field gradients. That acceleration would have the potential to wash out completely the fragile SN effect.

\subsubsection{Proposed Indirect Tests of SN Equation}

Indirect $\mathrm{SN}$ effects have been predicted for optomechanics systems which are comparably massive and on the verge to be quantum, see Fig. 4. Such effects are very small, can be overwhelmed by noise effects in the experiments, but can be done on the table-top. Therefore these tests represent a serious experimental challenge, while proposed to be possible with available technology. Two optomechanics experimental cases and the study of the SN dynamics in non-linear optics analogs are mentioned:

A. SN rotation of squeezed states The mechanical motion of an optomechanical system, clamped or levitated, is squeezed. Quantum squeezing of clamped optomechanics has been realised experimentally already, while a classical analog has been demonstrated for a levitated system. An optical homodyne detection of both field quadratures of the mechanical state is plotted and shows the cigar-shaped state, see in Fig. 6 left. The SN equation predicts an extra rotation of the squeezed phase-space distribution [73].

B. SN energy shifts of mechanical harmonic oscillator A further theoretical study [74] predicts SN related shifts of the Eigenenergy levels of the quantum harmonic oscillator describing the optomechanical system, see for an illustration of the multiple energy shift effects the Fig. 6 right. There different effects for the so-called wide and narrow wavefunction regimes are predicted for the situations that the spatial extent of the centre of mass motion wavefunction is larger (wide wavefunction regime) or smaller (narrow wavefunction regime) than the physical size of the massive object. A detailed experimental scenario has been worked out and awaits its realisation in an actual laboratory. 

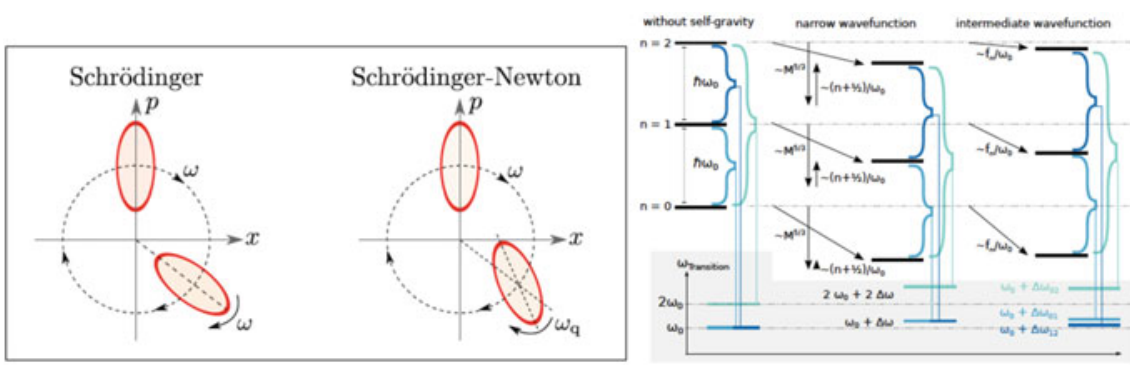

Fig. 6 Indirect Tests of the Schrödinger-Newton equation: Left Panel: Phase space plot of mechanical squeezed state with extra rotation of state distribution according to the SN effect. Left side: according to standard quantum mechanics, both the vector $(\langle x\rangle,\langle p\rangle)$ and the uncertainty ellipse of a Gaussian state for the centre of mass (CM) of a macroscopic object rotate clockwise in phase space, at the same frequency $\omega=\omega_{C M}$. Right side: according to the CM Schrödinger-Newton equation, $(\langle x\rangle,\langle p\rangle)$ still rotates at $\omega_{C M}$, but the uncertainty ellipse rotates at $\omega_{q}=\left(\omega_{C M}^{2}+\omega_{S N}^{2}\right)^{2}$. Picture taken from [73]. Right Panel: Schematic overview of the effect of the Schrödinger-Newton equation on the spectrum. The top part shows the first three energy eigenvalues and their shift due to the first order perturbative expansion of the Schrödinger-Newton potential. The bottom part shows the resulting spectrum of transition frequencies. In the narrow wavefunction regime (middle part), all energy levels are shifted down by an n-independent value minus an $n$-proportional contribution that scales with the inverse trap frequency. In the intermediate regime, where the wavefunction width becomes comparable to the localization length scale of the nuclei, this $n$-proportionality does no longer hold, leading to a removal of the degeneracy in the spectrum. Picture and caption taken from [74]

C. Non-linear optics simulation of the SN equation Specific delocalised nonlinearities in optical systems, typically just a piece of glass with a large refractive index, show a very similar type of dynamics for the propagation of light though that system if compared to SN dynamics. The analog holds at least in (1+1) space-time dimensions. The analog provides an interesting option to study the dynamics of the $\mathrm{SN}$ equation in a parameter regime complementary to numeric simulations. Some experiments have been already performed $[75,76]$ to study cosmological settings of the SN equation such as exotic Boson stars. The main question remains, what can we ultimately learn from optics analog experiments. Do we really learn about gravity? No, but we learn about the formal analog dynamics which is hard to calculate or simulate otherwise.

\subsection{Gravitational Decoherence Effects}

Tests of gravitational decoherence are based on the the straight-forward approach to generate a spatial superposition state (or any other non-classical state) of a massive particle and test if such a state decoheres according to (classical or quantum) gravity. Clearly, the experimental challenge is the preparation of such a state of sufficient mass. Typical experiments involve matterwave interferometers and quantum 
optomechanics. While the larges mass is given again by molecule interferometrysome of the effects (such as time dilation) are more promising to be tested in smaller mass systems such as cold atom interferometry, as those can be prepared in larger size superposition states to pick up a larger dephasing or decoherence effect. While on first sight it appears that only massive systems can be used for the test, we know that GR effects also exist for photons [77].

A. Gravitational decoherence affecting superpositions One of the proposed effects is by GR time dilation [78, 79], which is picked up as a dephasing effect for a matterwave interferometer for the propagation of the wavefunction along the two different arms - ultimately resulting in a reduction of the visibility of the interference pattern. The effect has been predicted to scale with the number of all internal degrees of freedom, which are involved in the energy-momentum tensor on the right hand side of Einstein's equations and therefore to affect the spacetime curvature and therefore gravity.

Atom interferometry tests, profiting from the high control on the centre of mass motion of cold atoms, e.g. in $10 \mathrm{~m}$ fountain and with sensitively on the verge of $10^{-19}$, of the time delation effect appear most promising at the moment, while the theoretical details of the effect are still debated. As a universal decoherence effect to explain the evident macroscopic quantum to classical transition, it is clear that that time dilation decoherence should it exist is weaker by many order of magnitude than know environmental effects such as decoherence due to collisions by an even very diluted background gas [80], which leaves the usefulness of the GR effect in question.

To be more precise, each (internal) degree of freedom of the particle is regarded as a clock running at a typical frequency, but depending via GR time dilation on the local gravitational environment. Then each single clock if separated between the two different paths of an interferometer will be sensitive to the relative duration of time and therefore dephase. This experiment has been realised as a proof of principle experiment with atomic chips [81], where the much larger spatial separation in other atomic interferometers [17] will help to improve the sensitivity to observe the predicted effect to test whether GR time dilation can be regarded as a universal source of decoherence to explain the macroscopic quantum to classical transition of physical systems, ultimately to explain the existence of the classical world.

B. Gravitational effect in dynamical reduction models Dynamical reduction or collapse models have been formulated to explain the quantum to classical transition on a fundamental level and in complement to decoherence models [14]. While the physics reason for the collapse to occur is explained by the existence of a universal classical and random noise field, the physics origin of that field is still debated. Gravity to be a candidate for the collapse field has to fulfil that two conditions of being classical and random. While the classicality is more straight forward, the implementation of a generic stochastic version of gravity represents a challenge. Some attempts have been undertaken and can also been seen as a stochastic modification of the Schrödinger-Newton equation, which was discussed in Sect. 3.1 [34, 82-84]. Tests of such gravity collapse models follow the same logic as tests of collapse models and in general a set of parameters has to be fulfilled. For more details related to 


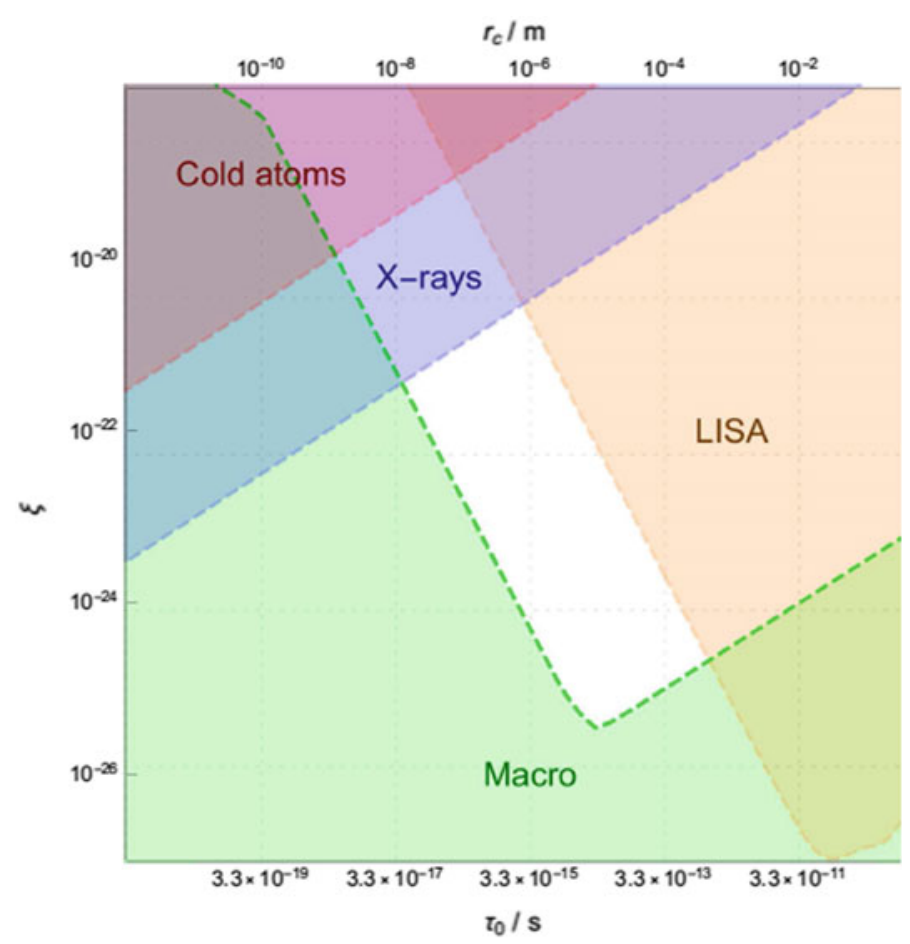

Fig. 7 Parameter map for gravity induced collapse models: $\left(\xi, r_{C}\right)$ or equivalently $\left(\xi, \tau_{0}\right)$ parameter diagram of the gravity-induced collapse model. The white area is the allowed region. The blue shaded region (X-rays) is excluded by data analysis of X-rays measurements. The orange shaded region (LISA) is excluded from data analysis of LISA Pathfinder. The green shaded region (Macro) is an estimate of the region excluded by the requirement that the collapse is strong enough to localize macroscopic objects. Note that $\mathrm{X}$-ray measurements sample the high frequency region of the spectrum $\left(10^{18} \mathrm{~Hz}\right)$ and would disappear if the noise correlator has a cutoff below such frequencies, which is plausible. In such a case, the stronger upper bound on the left part of the plane is given by data analysis with cold atom experiments (Cold atoms) [35]. Picture and caption has been taken from [84]

experimental test we refer to [84], where the Fig. 7) has been taken from. While the bounds on gravity collapse models in Fig. 7 relate to experiments already done, future experiments proposed to close the raining gap in the parameter plot involve those to generate large and massive quantum superpositions [30-32, 85]. Such experiments are currently under development in the laboratories.

C. Gravity induced collapse of the wavefunction Other ideas which are less related to the formalism of collapse models, but do explain the collapse of the wavefunction according to gravity are those independently by Diosí [86] and Penrose [87]. The best way to test those models is by large mass matterwave interferometry, where the mass has to be beyond the presently reached limit of molecule interferometry by many orders of magnitude. This means to test such models requires to preparation 
of large masses in non-classical states and optomechanical or magnetomechanical systems look most promising for the test [88-90]. Proposed experiments along those lines involve [31, 91].

D. Competing effects for matterwave interferometry In order to be able to see such gravity effects and how they collapse or decohere the wavefunction in matterwave based experiments all competing environmental decoherence processes have to be suppressed, which is the major experimental challenge in order to perform the experiments. Dominating decoherence effects are due to collisions with background gas, collisional decoherence [92] and the effects because of exchange of thermal radiation between the quantum system and the environment [30, 85]. Magnetic levitation of superconducting microparticles by definition avoids all effects related to internal temperature radiation as the experiment is cryogenic and on top of that all noises related to lasers are removed as well [31] which represents a huge advantage compared to optomechanics test. Further vibrations set serious constraints to all mechanics based test of wavefunction collapse and gravity.

E. The case for space Ultimately a test of gravity decoherence and gravity induced collapse of the wavefunction would benefit from large masses of the particles in superposition states as well as long lifetimes of those superposition states in order to observe the extremely weak effects. The space proposal on macroscopic quantum resonators (MAQRO) [33] would be able to fulfil such all those conditions. A community has started to work towards such a test in space and to propose a related mission.

\subsection{The Gravity of a Quantum State-Revisited}

What gravitational field is generated by a massive quantum system in a spatial superposition? Despite decades of intensive theoretical and experimental research, we still do not know the answer. On the experimental side, the difficulty lies in the fact that gravity is weak and requires large masses to be detectable. However, it becomes increasingly difficult to generate spatial quantum superpositions for increasingly large masses, in light of the stronger environmental effects on such systems. Clearly, a delicate balance between the need for strong gravitational effects and weak decoherence should be found. We show that such a trade off could be achieved in an optomechanics scenario that allows to witness whether the gravitational field generated by a quantum system in a spatial superposition is in a coherent superposition or not. We estimate the magnitude of the effect and show that it offers perspectives for observability.

Quantum field theory is one of the most successful theories ever formulated. All matter fields, together with the electromagnetic and nuclear forces, have been successfully embedded in the quantum framework. They form the standard model of elementary particles, which not only has been confirmed in all advanced accelerator facilities, but has also become an essential ingredient for the description of the universe and its evolution. 
In light of this, it is natural to seek a quantum formulation of gravity as well. Yet, the straightforward procedure for promoting the classical field as described by general relativity, into a quantum field, does not work. Several strategies have been put forward, which turned into very sophisticated theories of gravity, the most advanced being string theory and loop quantum gravity. Yet, none of them has reached the goal of providing a fully consistent quantum theory of gravity.

At this point, one might wonder whether the very idea of quantizing gravity is correct [59-64, 66, 66-68, 93, 94]. At the end of the day, according to general relativity, gravity is rather different from all other forces. Actually, it is not a force at all, but a manifestation of the curvature of spacetime, and there is no obvious reason why the standard approach to the quantization of fields should work for spacetime as well. A future unified theory of quantum and gravitational phenomena might require a radical revision not only of our notions of space and time, but also of (quantum) matter. This scenario is growing in likeliness [95-97].

From the experimental point of view, it has now been ascertained that quantum matter (i.e. matter in a genuine quantum state, such as a coherent superposition state) couples to the Earth's gravity in the most obvious way. This has been confirmed in neutron, atom interferometers and used for velocity selection in molecular interferometry. However, in all cases, the gravitational field is classical, i.e. it is generated by a distribution of matter (the Earth) in a fully classical state. Therefore, the plethora of successful experiments mentioned above does not provide hints, unfortunately, on whether gravity is quantum or not.

In a recent paper [98], we discuss an approach where a quantum system is forced in the superposition of two different positions in space, and its gravitational field is explored by a probe (Fig. 8). Using the exquisite potential for transduction offered by optomechanics, we can in principle witness whether the gravitational field is the

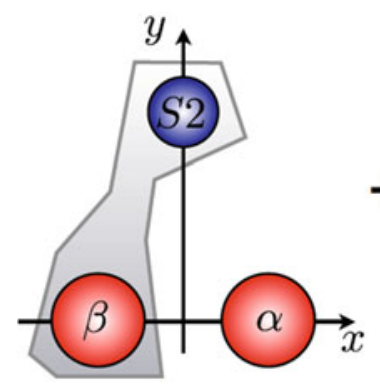

(a)

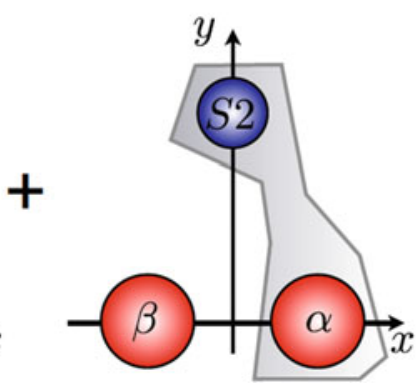

(b)

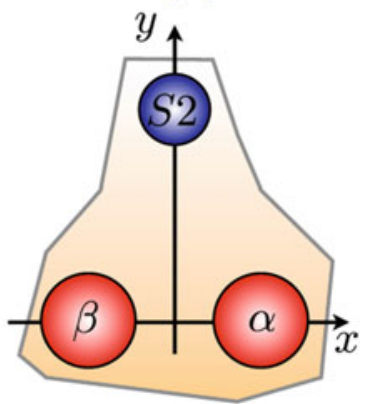

Fig. 8 Schematic representation of the two-body setup. S1 is prepared in a spatial superposition along the $x$ direction (red balls). $\mathrm{S} 2$ is initially prepared in a localized wavepacket (blue ball), and it probes the gravitational field generated by S1. a The gravitational field acting on S2 is a linear combination of gravitational fields produced by $\mathrm{S} 1$ being in a superposed state. $\mathbf{b}$ The semi-classical treatment of gravity, where the gravitational field acting on S2 is that produced by a total mass $m_{1}$ with density $\frac{1}{2}\left(|\alpha(r)|^{2}+|\beta(r)|^{2}\right)$ 
superposition of the two gravitational fields associated to the two different states of the system, or not. The first case amounts to a quantum behavior of gravity, the second to a classical-like one. We have illustrated the dynamics of an optomechanical system probing the gravitational field of a massive quantum system in a spatial superposition. Two different dynamics are found whether gravity is treated quantum mechanically or classically. Here, we propose two distinct methods to infer which of the two dynamics rules the motion of the quantum probe, thus discerning the intrinsic nature of the gravitational field. Such methods will be then eventually able to falsify one of the two treatments of gravity. A similar proposal has been made for angular superpositions [99].

The considered setup is formed of two systems interacting gravitationally. All nongravitational interactions are considered, for all practical purposes, negligible. The first system (S1) has a mass $m_{1}$, and it is initially prepared in a spatial superposition along the $x$ direction. Its wave-function is $\psi\left(r_{1}\right)=\frac{1}{\sqrt{2}}\left(\alpha\left(r_{1}\right)+\beta\left(r_{1}\right)\right)$, where $\alpha\left(r_{1}\right)$ and $\beta\left(r_{1}\right)$ are sufficiently well localized states in position, far from each other in order to prevent any overlap. Thus, we can consider them as distinguishable (in a macroscopic sense), and we approximate $\langle\alpha \mid \beta\rangle \simeq 0$. The second system (S2) will serve as a point-like probe of the gravitational field generated by $\mathrm{S} 1$, it has mass $m_{2}$ and state $\phi\left(r_{2}\right)$. The state $\phi\left(r_{2}\right)$ is initially assumed to be localized in position and centered along the $y$ direction [cf. Fig. 8]. The question we address is: which is the gravitational field, generated by the quantum superposition of $S 1$, that $S 2$ experiences? We probe the following two different scenarios.

Quantum Gravity Scenario. Although we do not have a quantum theory of gravity so far, one can safely claim that, regardless of how it is realized, it would manifest in $\mathrm{S} 1$ generating a superposition of gravitational fields. As discussed in the introduction, the assessment of this property precedes the quest to ascertain the existence of the graviton and the characterization of its properties, at least as far as the static, lowenergy, non-relativistic regime we are considering is concerned. Linearity is the very characteristic trait of quantum theory, and one expects it to be preserved by any quantum theory of gravity.

The reaction of $\mathrm{S} 2$ is then to go in a superposition of being attracted towards the region where $|\alpha\rangle$ sits and where $|\beta\rangle$ does. The final two-body state will have the following entangled form

$$
\Psi_{\mathrm{QG}}^{\mathrm{final}}\left(r_{1}, r_{2}\right)=\frac{\alpha\left(\mathbf{r}_{1}\right) \phi_{\alpha}\left(\mathbf{r}_{2}\right)+\beta\left(\mathbf{r}_{1}\right) \phi_{\beta}\left(\mathbf{r}_{2}\right)}{\sqrt{2}},
$$

where $\phi_{\alpha}\left(\mathbf{r}_{2}\right)\left(\phi_{\beta}\left(\mathbf{r}_{2}\right)\right)$ represents the state of S2 attracted towards the region where $|\alpha\rangle(|\beta\rangle)$ rests. The motion in each branch of the superposition is produced by the potential

$$
\hat{V}_{\gamma}\left(\hat{r}_{2}\right)=-G m_{2} \int \mathrm{d} r_{1} \frac{\rho_{\gamma}\left(\mathbf{r}_{1}\right)}{\left|r_{1}-\hat{r}_{2}\right|}, \quad(\gamma=\alpha, \beta) .
$$


where $\rho_{\gamma}\left(\mathbf{r}_{1}\right)$ is the mass density of $\mathrm{S} 1$, centred in $\left\langle\hat{r}_{1}\right\rangle_{\gamma}=\left\langle\gamma\left|\hat{r}_{1}\right| \gamma\right\rangle$. We assume that $\mathrm{S} 1$ does not move appreciably during the time of the experiment (also quantum fluctuations can be neglected); clearly, such a situation can be assumed only as long as the S1 superposition lives. We further assume that its mass density is essentially spheric, so that the gravitational interaction can be approximated by

$$
\hat{V}_{\gamma}\left(\hat{r}_{2}\right) \approx-\frac{G m_{1} m_{2}}{\left|\left\langle\hat{\mathbf{r}}_{1}\right\rangle_{\gamma}-\hat{\mathbf{r}}_{2}\right|}, \quad(\gamma=\alpha, \beta) .
$$

Semiclassical Gravity Scenario. The second scenario sees gravity as fundamentally classical. In this case, it is not clear which characteristics one should expect from the gravitational field generated by a superposition. However, in analogy with classical mechanics, one can assume that is the mass density $\rho\left(\mathbf{r}_{1}\right)=\left(\rho_{\alpha}\left(\mathbf{r}_{1}\right)+\rho_{\beta}\left(\mathbf{r}_{1}\right)\right) / 2$ of the system in superposition that produces the gravitational field. This is also what is predicted by the Schrödinger-Newton equation (see Sect. 3). The final two-body state will be of the form

$$
\Psi_{\mathrm{CG}}^{\mathrm{final}}\left(r_{1}, r_{2}\right)=\frac{\alpha\left(\mathbf{r}_{1}\right)+\beta\left(\mathbf{r}_{1}\right)}{\sqrt{2}} \phi\left(r_{2}\right),
$$

where the difference with Eq. (9) is clear. The gravitational potential becomes

$$
\hat{V}_{\mathrm{cl}}\left(\hat{r}_{2}\right) \approx \frac{1}{2} \sum_{\gamma=\alpha, \beta} \hat{V}_{\gamma}\left(\hat{r}_{2}\right),
$$

where $\hat{V}_{\gamma}\left(\hat{r}_{2}\right)$ can be eventually approximated as in Eq. (11).

Experimental progress with levitated mechanical systems makes is possible to reach a parameter regime to experimentally resolve the difference between the quantum and semiclassical scenarios as shown in our paper [98]. Other interferometric $[100,101]$ and non-interferometric [102] tests of the nature of gravity have been proposed. They are based on the detection of entanglement between two probes, respectively coupled to two different massive systems, which interact through gravity (NV center spins for [100] and cavity fields for [102]). Clearly, to have such entanglement, each of the three couples of interconnected systems (probe 1, system 1, system 2 and probe 2) there considered needs to be entangled on their own. Moreover, the entanglement between the two massive systems is inevitably small due to its gravitational nature. Conversely, our proposal benefits from having only a single massive system involved in the interconnection, which reduces correlation losses. In addition, we provide a second method for discerning the nature of gravity: the individuation of a second peak in the DNS. The latter does not rely on delicate measurements of quantum correlations but can be assessed through standard optomechanical detection schemes. 


\subsection{Concluding Remarks on Testing the Interplay of Quantum Mechanics and Gravity in the Low Energy Regime}

While matterwave interferometer experiments have been performed in the low mass regime, see Fig. 4, the higher mass range, all the way up to milligram masses is unexplored by any experiment and especially not by any quantum experiment. Optomechanical devices and especially levitated particles are able to bridge this enormous mass gap; being in a quantum mechanical state and very massive at the same time. Levitated mechanical systems hold promise to test new physics in that new mass range. A variety of theoretical proposals and ideas for the interplay between quantum mechanics and gravity will become testable in this very mass range. The study of gravitational decoherence, the Schrödinger-Newton equation and the gravity of a quantum state provide concrete routes for experimental exploration.

\section{Simulation of the Stern Gerlach Experiment Using Wigner Functions}

The Stern-Gerlach (SG) experiment [103] is a seminal example of a quantum experiment involving coupling between internal and external degrees of freedom. In this experiment, an electron or nuclear spin interacts with a spatially inhomogeneous magnetic field through the magnetic Zeeman interaction. The outcome of the SternGerlach experiment is, of course, "well-known": an incident molecular beam of particles with spin-1/2 is separated by the inhomogeneous magnetic field into two beams, each corresponding to particles with well-defined spin angular momenta along the field direction. But how does this separation happen in detail, on the level of the spatial quantum state?

In a recent article [104] we used an extended Wigner function (EWF) which includes the presence of internal degrees of freedom in the propagating particle, and the coupling of those internal degrees of freedom to inhomogeneous external fields (Fig. 9).

The Wigner function $W(x, p)$ is a joint quasi-probability density function defined over the combined domains of the spatial coordinate(s) $x$ and its associated momentum (momenta) $p$. It is defined as a Weyl integral transform of the density operator $\hat{\rho}=|\psi\rangle\langle\psi|$, of the following form:

$$
W(x, p)=\frac{1}{h} \int e^{-\frac{i p s}{\hbar}}\left\langle x+\frac{s}{2}|\hat{\rho}| x-\frac{s}{2}\right\rangle d s .
$$

Consider a particle with a finite number of internal quantum states. In the discussion below, we refer to these internal states as "spin states", although the same formalism applies to non-spin degrees of freedom, such as quantized rotational and 

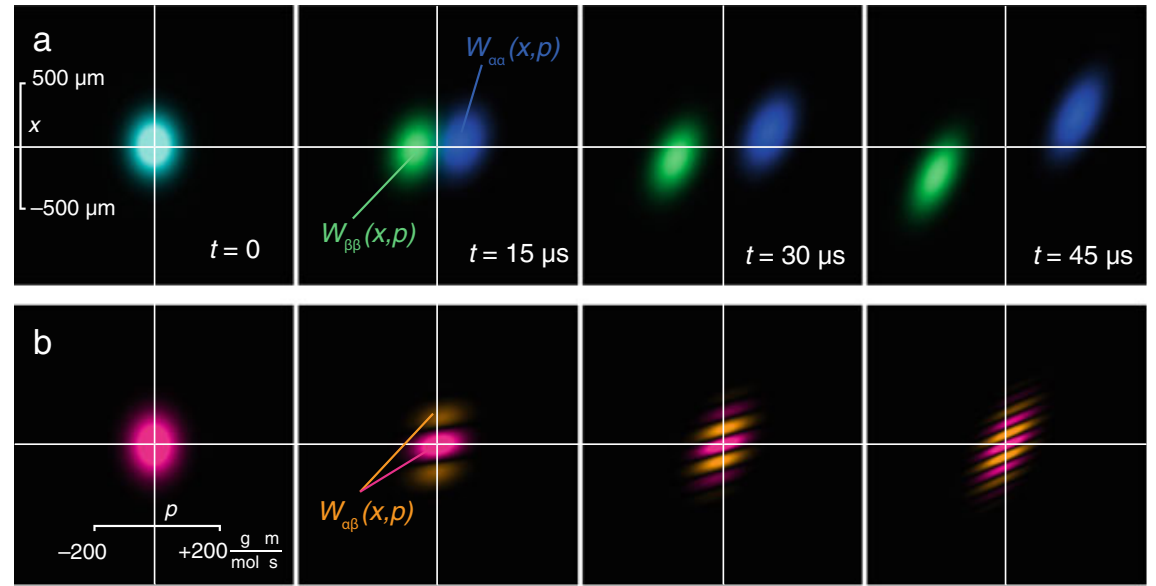

Fig. 9 a Evolution of $W_{\alpha \alpha}$ and $W_{\beta \beta}$ under the influence of a magnetic field gradient in a SternGerlach experiment on $A g$ atoms in a field gradient of $10 \mathrm{G} \mathrm{m} \mathrm{m}^{-1}$, moving at a velocity of $550 \mathrm{~m} / \mathrm{s}$ (rms velocity at an oven temperature of $1300 \mathrm{~K}$ ). b Evolution of the real part of the off-diagonal element $W_{\alpha \beta}$, assuming a coherent state initially polarised along the $x$-axis. The strength of the magnetic field gradient has been reduced by a factor of $5 \times 10^{4}$ compared to $\mathrm{A}$ in order to make the spatial modulation visible. The shearing of the fine structure of the Wigner function represents decoherence. Figure and capture taken from Ref. [104]

vibrational states. We extend the Wigner function by combining it with the density operator formalism commonly used in the quantum description of magnetic resonance. The definition of the Wigner function is extended by projecting the density operator onto the spin-state specific position state $|x, \eta\rangle$, where $\eta=\alpha, \beta, \ldots$ denotes the spin state. This results in a Wigner probability density matrix $W_{\eta \xi}(x, p)$, whose elements depend parametrically on the positional variables and their associated momenta:

$$
W_{\eta \xi}(x, p)=\frac{1}{h} \int e^{-\frac{i p s}{\hbar}}\left\langle x+\frac{s}{2}, \eta|\hat{\rho}| x-\frac{s}{2}, \xi\right\rangle d s .
$$

This means the extended Wigner function can be used to directly simulate the SG experiment. In the Stern-Gerlach experiment, a beam of spin-1/2 particles is exposed to a lateral magnetic field gradient. We define the axis of the molecular beam apparatus as $z$, and assume that the magnetic field varies in the transverse $x$ direction. The potential energy part of the Hamiltonian in the presence of an external magnetic field $\mathbf{B}$ is then given by

$$
U(\hat{\mathbf{S}}, x)=-\hbar \gamma \mathbf{B}(\mathbf{x}) \cdot \hat{\mathbf{S}}
$$

The original magnet design used by Stern and Gerlach [103] produces divergent magnetic field lines at the location of the beam. This corresponds to a biaxial magnetic 
field gradient tensor, requiring two spatial dimensions to be included in the Wigner function. To avoid this complication, we use a different arrangement, in which the magnetic field gradient is uniaxial. In this case, the magnetic field lines are all parallel, but vary in density in the direction perpendicular to the magnetic field itself. Magnetic fields of this type occur in quadrupole polarisers.

We assume the magnetic field points along the $y$-axis, and varies linearly in magnitude along the $x$-axis, $\mathbf{B}(\mathbf{x}, \mathbf{y}, \mathbf{z})=\left(\mathbf{B}_{\mathbf{y} \mathbf{0}}+\mathbf{x} \mathbf{G}_{\mathbf{x y}}\right) \mathbf{e}_{\mathbf{y}}$, where $B_{y 0}$ is the magnetic field at $x=0$, and $G_{x y}=\partial B_{y} / \partial x$. This field is fully consistent with Maxwell's equations, since it satisfies $\nabla \cdot \mathbf{B}=\mathbf{0}$. The field gradient has only a single non-zero cartesian component $\nabla \mathbf{B}=\mathbf{G}_{\mathbf{x y}} \mathbf{e}_{\mathbf{x}} \mathbf{e}_{\mathbf{y}}$. We choose the spin states $|\alpha\rangle$ and $|\beta\rangle$ as the eigenstates of $\hat{S}_{y}$, such that the matrix elements of the potential part of the Hamiltonian are

$$
\begin{array}{ll}
U_{\alpha \alpha}(x)=-\frac{\gamma \hbar}{2} B_{y}(x) & U_{\alpha \beta}(x)=0 \\
U_{\beta \alpha}(x)=0 & U_{\beta \beta}(x)=+\frac{\gamma \hbar}{2} B_{y}(x) .
\end{array}
$$

The resulting equations of motion for the EWF matrix elements are given in the SI.

In its original form, the Stern-Gerlach experiment was conducted on a beam of $\mathrm{Ag}$ atoms emanating from an oven at a temperature of about $1300 \mathrm{~K}$. The magnetic field gradient was of the order of $10 \mathrm{G} / \mathrm{cm}$ over a length of $3.5 \mathrm{~cm}$ [105]. For simplicity, we ignore the nuclear spin of $\mathrm{Ag}$, and treat the atoms as (electron) spin $1 / 2$ particles. In the case of magnetic fields larger than the hyperfine splitting (about $610 \mathrm{G}$ in the case of $\mathrm{Ag}$ ), this is a good approximation, since the nuclear and the electron spin states are essentially decoupled. The root mean square velocity of $\mathrm{Ag}$ atoms $1300 \mathrm{~K}$ is approximately $550 \mathrm{~m} / \mathrm{s}$. After leaving the oven, the Ag atoms are collimated by a pair of collimation slits $30 \mu \mathrm{m}$ wide and separated by $3 \mathrm{~cm}$. The longitudinal momentum of the silver atoms is approximately $6 \times 10^{4} \mathrm{~g} \mathrm{~mol}^{-1} \mathrm{~ms}^{-1}$. The collimation aspect ratio of 1:1000 therefore results in a transverse momentum uncertainty of $\Delta p=60 \mathrm{~g} \mathrm{~mol}^{-1} \mathrm{~ms}^{-1}$, which corresponds to a $30 \mu \mathrm{m}$ wide beam with a transverse coherence length of about $l_{c}=h / \Delta p \approx 7 \mathrm{~nm}$.

An unpolarised beam entering the magnetic field gradient is represented by a unity spin density matrix, such that $W_{\alpha \alpha}(t=0)=W_{\beta \beta}(t=0)=W_{0}(x, p)$, where the initial state $W_{0}(x, p)$ is a two-dimensional normalised Gaussian function centred at $(x, p)=(0,0)$, with widths given by coherence length $l_{c}$ and the beam width $\Delta x$ (cf. SI). The off-diagonal Wigner functions vanish: $W_{\alpha \beta}=W_{\beta \alpha} \equiv 0$, and the diagonal ones can be obtained in closed form by integrating the equations of motion (cf. SI).

In conclusion, the SG magnet can be used as coherent beam splitter, but the original experiment did not do the recombination or any other protocol to demonstrate the quantum correlation. When a coherent superposition spin state is provided at the SG input then a coherent spatial superposition of the centre of mass motion of the particle can be achieved. This has been finally demonstrated by the group of Ron Folman [106] for the case of atom interferometry and is used as central ingredient for a recent proposal of the generation of macroscopic quantum superposition [100, 101]. 
Acknowledgements I would like to thank my research team members at Southampton and external collaborators for joint work on testing fundamental physics with mechanical systems in table-top experiments over the past ten years. We are getting there.

\section{References}

1. J.P. Toennies, H. Schmidt-Böcking, B. Friedrich, J.C. Lower, Otto Stern (1888-1969): The founding father of experimental atomic physics. Ann. Phys. 523(12), 1045-1070 (2011)

2. S. Deachapunya, P.J. Fagan, A.G. Major, E. Reiger, H. Ritsch, A. Stefanov, H. Ulbricht, M. Arndt, Slow beams of massive molecules. Euro. Phys. J. D 46(2), 307-313 (2008)

3. P. Asenbaum, S. Kuhn, S. Nimmrichter, U. Sezer, M. Arndt, Cavity cooling of free silicon nanoparticles in high vacuum. Nat. Commun. 4(1), 1-7 (2013)

4. J. Millen, T.S. Monteiro, R. Pettit, A.N. Vamivakas, Optomechanics with levitated particles. Rep. Prog. Phys. 83(2), 026401 (2020)

5. A. Ashkin, Optical trapping and manipulation of neutral particles using lasers: A reprint volume with commentaries (2006)

6. J. Millen, P.Z.G. Fonseca, T. Mavrogordatos, T.S. Monteiro, P.F. Barker, Cavity cooling a single charged levitated nanosphere. Phys. Rev. Lett. 114(12), 123602 (2015)

7. D.C. Moore, A.D. Rider, G. Gratta, Search for millicharged particles using optically levitated microspheres. Phys. Rev. Lett. 113(25), 251801 (2014)

8. C. Timberlake, G. Gasbarri, A. Vinante, A. Setter, H. Ulbricht, Acceleration sensing with magnetically levitated oscillators above a superconductor. Appl. Phys. Lett. 115(22), 224101 (2019)

9. Y.Y. Fein, P. Geyer, P. Zwick, F. Kialka, S. Pedalino, M. Mayor, S. Gerlich, M. Arndt, Quantum superposition of molecules beyond $25 \mathrm{kDa}$. Nat. Phys. 15(12), 1242-1245 (2019)

10. S. Nimmrichter, K. Hornberger, Macroscopicity of mechanical quantum superposition states. Phys. Rev. Lett. 110(16), 160403 (2013)

11. A. Bassi, G. Ghirardi, Dynamical reduction models. Phys. Rep. 379(5-6), 257-426 (2003)

12. G.C. Ghirardi, A. Rimini, T. Weber, Unified dynamics for microscopic and macroscopic systems. Phys. Rev. D 34(2), 470 (1986)

13. G.C. Ghirardi, P. Pearle, A. Rimini, Markov processes in Hilbert space and continuous spontaneous localization of systems of identical particles. Phys. Rev. A 42(1), 78 (1990)

14. A. Bassi, K. Lochan, S. Satin, T.P. Singh, H. Ulbricht, Models of wave-function collapse, underlying theories, and experimental tests. Rev. Mod. Phys. 85(2), 471 (2013)

15. S.L. Adler, Lower and upper bounds on CSL parameters from latent image formation and IGM heating. J. Phys. A: Math. Theor. 40(12), 2935 (2007)

16. S. Eibenberger, S. Gerlich, M. Arndt, M. Mayor, J. Tüxen, Matter-wave interference of particles selected from a molecular library with masses exceeding $10000 \mathrm{amu}$. Phys. Chem. Chem. Phys. 15(35), 14696-14700 (2013)

17. T. Kovachy, P. Asenbaum, C. Overstreet, C.A. Donnelly, S.M. Dickerson, A. Sugarbaker, J.M. Hogan, M.A. Kasevich, Quantum superposition at the half-metre scale. Nature 528(7583), 530-533 (2015)

18. K.C. Lee, M.R. Sprague, B.J. Sussman, J. Nunn, N.K. Langford, X.M. Jin, T. Champion, P. Michelberger, K.F. Reim, D. England, D. Jaksch, Entangling macroscopic diamonds at room temperature. Science 334(6060), 1253-1256 (2011)

19. M. Armano, H. Audley, J. Baird, P. Binetruy, M. Born, D. Bortoluzzi, E. Castelli, A. Cavalleri, A. Cesarini, A.M. Cruise, K. Danzmann, Beyond the required LISA free-fall performance: new LISA Pathfinder results down to $20 \mathrm{~Hz}$. Phys. Rev. Lett. 120(6), 061101 (2018)

20. M. Armano, H. Audley, G. Auger, J.T. Baird, M. Bassan, P. Binetruy, M. Born, D. Bortoluzzi, N. Brandt, M. Caleno, L. Carbone, Sub-femto-g free fall for space-based gravitational wave observatories: LISA pathfinder results. Phys. Rev. Lett. 116(23), 231101 (2016) 
21. T. Kovachy, J.M. Hogan, A. Sugarbaker, S.M. Dickerson, C.A. Donnelly, C. Overstreet, M.A. Kasevich, Matter wave lensing to picokelvin temperatures. Phys. Rev. Lett. 114(14), 143004 (2015)

22. S.L. Adler, A. Vinante, Bulk heating effects as tests for collapse models. Phys. Rev. A 97(5), 052119 (2018)

23. K. Piscicchia, A. Bassi, C. Curceanu, R.D. Grande, S. Donadi, B.C. Hiesmayr, A. Pichler, CSL collapse model mapped with the spontaneous radiation. Entropy 19(7), 319 (2017)

24. A. Vinante, M. Bahrami, A. Bassi, O. Usenko, G.H.C.J. Wijts, T.H. Oosterkamp, Upper bounds on spontaneous wave-function collapse models using millikelvin-cooled nanocantilevers. Phys. Rev. Lett. 116(9), 090402 (2016)

25. M. Torol, G. Gasbarri, A. Bassi, Colored and dissipative continuous spontaneous localization model and bounds from matter-wave interferometry. Phys. Lett. A 381(47), 3921-3927 (2017)

26. M. Carlesso, A. Bassi, Current tests of collapse models: How far can we push the limits of quantum mechanics? in Quantum Information and Measurement (Optical Society of America, 2019, April), pp. S1C-3

27. M. Toroš, A. Bassi, Bounds on quantum collapse models from matter-wave interferometry: Calculational details. J. Phys. A: Math. Theoretical 51(11), 115302 (2018)

28. C. Gardiner, P. Zoller, Quantum Noise: A Handbook of Markovian and Non-Markovian Quantum Stochastic Methods with Applications to Quantum Optics Vol. 56 (Springer Science and Business Media, 2004)

29. H.P. Breuer, F. Petruccione, The Theory of Open Quantum Systems (Oxford University Press, Oxford, 2002)

30. J. Bateman, S. Nimmrichter, K. Hornberger, H. Ulbricht, Near-field interferometry of a freefalling nanoparticle from a point-like source. Nat. Commun. 5(1), 1-5 (2014)

31. H. Pino, J. Prat-Camps, K. Sinha, B.P. Venkatesh, O. Romero-Isart, On-chip quantum interference of a superconducting microsphere. Quantum Sci. Technol. 3(2), 025001 (2018)

32. C. Wan, M. Scala, G.W. Morley, A.A. Rahman, H. Ulbricht, J. Bateman, P.F. Barker, S. Bose, M.S. Kim, Free nano-object Ramsey interferometry for large quantum superpositions. Phys. Rev. Lett. 117(14), 143003 (2016)

33. R. Kaltenbaek, M. Aspelmeyer, P.F. Barker, A. Bassi, J. Bateman, K. Bongs, S. Bose, C. Braxmaier, Brukner, Christophe, B., Chwalla, M., Macroscopic quantum resonators (MAQRO): 2015 update. EPJ Quantum Technol. 3(1), 5 (2016)

34. M. Bahrami, A. Smirne, A. Bassi, Role of gravity in the collapse of a wave function: A probe into the diósi-penrose model. Phys. Rev. A 90(6), 062105 (2014)

35. B. Collett, P. Pearle, Wavefunction collapse and random walk. Found. Phys. 33(10), 14951541 (2003)

36. S.L. Adler, Stochastic collapse and decoherence of a non-dissipative forced harmonic oscillator. J. Phys. A: Math. Gen. 38(12), 2729 (2005)

37. K. Hornberger, S. Gerlich, P. Haslinger, S. Nimmrichter, M. Arndt, Colloquium: Quantum interference of clusters and molecules. Rev. Mod. Phys. 84(1), 157 (2012)

38. S. Nimmrichter, K. Hornberger, K. Hammerer, Optomechanical sensing of spontaneous wavefunction collapse. Phys. Rev. Lett. 113(2), 020405 (2014)

39. S. Bera, B. Motwani, T.P. Singh, H. Ulbricht, A proposal for the experimental detection of CSL induced random walk. Sci. Rep. 5(1), 1-10 (2015)

40. L. Diósi, Testing spontaneous wave-function collapse models on classical mechanical oscillators. Phys. Rev. Lett. 114(5), 050403 (2015)

41. D. Goldwater, M. Paternostro, P.F. Barker, Testing wave-function-collapse models using parametric heating of a trapped nanosphere. Phys. Rev. A 94(1), 010104 (2016)

42. M. Carlesso, M. Paternostro, H. Ulbricht, A. Vinante, A. Bassi, Non-interferometric test of the continuous spontaneous localization model based on rotational optomechanics. New J. Phys. 20(8), 083022 (2018)

43. M. Carlesso, A. Bassi, P. Falferi, A. Vinante, Experimental bounds on collapse models from gravitational wave detectors. Phys. Rev. D 94(12), 124036 (2016) 
44. W. Paul, Electromagnetic traps for charged and neutral particles. Rev. Mod. Phys. 62(3), 531 (1990)

45. A. Vinante, A. Pontin, M. Rashid, M. Torol, P.F. Barker, H. Ulbricht, Testing collapse models with levitated nanoparticles: Detection challenge. Phys. Rev. A 100(1), 012119 (2019)

46. R. Mishra, A. Vinante, T.P. Singh, Testing spontaneous collapse through bulk heating experiments: An estimate of the background noise. Phys. Rev. A 98(5), 052121 (2018)

47. M. Bilardello, S. Donadi, A. Vinante, A. Bassi, Bounds on collapse models from cold-atom experiments. Phys. A 462, 764-782 (2016)

48. A. Pontin, N.P. Bullier, M. Torol, P.F. Barker, Ultranarrow-linewidth levitated nano-oscillator for testing dissipative wave-function collapse. Phys. Rev. Res. 2(2), 023349 (2020)

49. B.R. Slezak, C.W. Lewandowski, J.F. Hsu, D’Urso, B., Cooling the motion of a silica microsphere in a magneto-gravitational trap in ultra-high vacuum. New J. Phys. 20(6), 063028 (2018)

50. D. Zheng, Y. Leng, X. Kong, R. Li, Z. Wang, X. Luo, J. Zhao, C.K. Duan, P. Huang, J. Du, Room temperature test of wave-function collapse using a levitated micro-oscillator (2019). arXiv preprint arXiv: 1907.06896

51. O. Romero-Isart, L. Clemente, C. Navau, A. Sanchez, J.I. Cirac, Quantum magnetomechanics with levitating superconducting microspheres. Phys. Rev. Lett. 109(14), 147205 (2012)

52. B. van Waarde, The lead zeppelin: a force sensor without a handle, Ph.D. Thesis, Leiden University (2016)

53. A. Vinante, P. Falferi, G. Gasbarri, A. Setter, C. Timberlake, H. Ulbricht, Ultralow mechanical damping with Meissner-levitated ferromagnetic microparticles. Phys. Rev. Appl. 13(6), 064027 (2020)

54. J. Prat-Camps, C. Teo, C.C. Rusconi, W. Wieczorek, O. Romero-Isart, Ultrasensitive inertial and force sensors with diamagnetically levitated magnets. Phys. Rev. Appl. 8(3), 034002 (2017)

55. M. Bahrami, A. Bassi, H. Ulbricht, Testing the quantum superposition principle in the frequency domain. Phys. Rev. A 89(3), 032127 (2014)

56. S. Sturm, F. Köhler, J. Zatorski, A. Wagner, Z. Harman, G. Werth, W. Quint, C.H. Keitel, K. Blaum, High-precision measurement of the atomic mass of the electron. Nature 506(7489), 467-470 (2014)

57. M. Weitz, A. Huber, F. Schmidt-Kaler, D. Leibfried, T.W. Hänsch, Precision measurement of the hydrogen and deuterium 1 S ground state Lamb shift. Phys. Rev. Lett. 72(3), 328 (1994)

58. E. Oelker, R.B. Hutson, C.J. Kennedy, L. Sonderhouse, T. Bothwell, A. Goban, D. Kedar, C. Sanner, J.M. Robinson, G.E. Marti, D.G. Matei, Demonstration of $4.8 \times 10^{-17}$ stability at 1 $\mathrm{s}$ for two independent optical clocks. Nat. Photon. 13(10), pp.714-719 (2019)

59. R. Penrose, On the gravitization of quantum mechanics 1: Quantum state reduction. Found. Phys. 44(5), 557-575 (2014)

60. L. Rosenfeld, On quantization of fields. Nuclear Phys. 40, 353-356 (1963)

61. S. Carlip, Is quantum gravity necessary? Class. Quantum Gravity 25(15), 154010 (2008)

62. C. Møller, Les théories relativistes de la gravitation. Colloques Internationaux CNRS 91(1) (1962)

63. D.N. Page, C.D. Geilker, Indirect evidence for quantum gravity. Phys. Rev. Lett. 47(14), 979 (1981)

64. K. Eppley, E. Hannah, The necessity of quantizing the gravitational field. Found. Phys. 7(1-2), 51-68 (1977)

65. N. Gisin, Stochastic quantum dynamics and relativity. Helv. Phys. Acta 62(4), 363-371 (1989)

66. J. Mattingly, Why Eppley and Hannah's thought experiment fails. Phys. Rev. D 73(6), 064025 (2006)

67. C. Kiefer, Why quantum gravity? in Approaches to Fundamental Physics (Springer, Berlin, Heidelberg, 2007), pp. 123-130

68. M. Albers, C. Kiefer, M. Reginatto, Measurement analysis and quantum gravity. Phys. Rev. D 78(6), 064051 (2008) 
69. D. Giulini, A. Großardt, Gravitationally induced inhibitions of dispersion according to the Schrödinger-Newton equation. Class. Quantum Gravity 28(19), 195026 (2011)

70. D. Giulini, A. Großardt, Gravitationally induced inhibitions of dispersion according to a modified Schrödinger-Newton equation for a homogeneous-sphere potential. Class. Quantum Gravity 30(15), 155018 (2013)

71. L. Diósi, Gravitation and quantum-mechanical localization of macro-objects. Phys. Lett. A 105(4-5), 199-202 (1984)

72. R. Penrose, Quantum computation, entanglement and state reduction. Philos. Trans. R. Soc. London. Ser. A: Math. Phys. Eng. Sci. 356(1743), 1927-1939

73. H. Yang, H. Miao, D.S. Lee, B. Helou, Y. Chen, Macroscopic quantum mechanics in a classical spacetime. Phys. Rev. Lett. 110(17), 170401 (2013)

74. A. Großardt, J. Bateman, H. Ulbricht, A. Bassi, Optomechanical test of the SchrödingerNewton equation. Phys. Rev. D 93(9), 096003 (2016)

75. R. Bekenstein, R. Schley, M. Mutzafi, C. Rotschild, M. Segev, Optical simulations of gravitational effects in the Newton-Schrödinger system. Nat. Phys. 11(10), 872-878 (2015)

76. T. Roger, C. Maitland, K. Wilson, N. Westerberg, D. Vocke, E.M. Wright, D. Faccio, Optical analogues of the Newton-Schrödinger equation and boson star evolution. Nat. Commun. 7(1), $1-8$ (2016)

77. M. Zych, F. Costa, I. Pikovski, T.C. Ralph, Č. Brukner, General relativistic effects in quantum interference of photons. Class. Quantum Gravity 29(22), 224010 (2012)

78. M. Zych, F. Costa, I. Pikovski, Č. Brukner, Quantum interferometric visibility as a witness of general relativistic proper time. Nat. Commun. 2, 505 (2011)

79. I. Pikovski, M. Zych, F. Costa, Č. Brukner, Universal decoherence due to gravitational time dilation. Nat. Phys. 11(8), 668-672 (2015)

80. M. Torol, A. Bassi, Bounds on quantum collapse models from matter-wave interferometry: Calculational details. J. Phys. A: Math. Theor. 51(11), 115302 (2018)

81. Y. Margalit, Z. Zhou, S. Machluf, D. Rohrlich, Y. Japha, R. Folman, A self-interfering clock as a "which path" witness. Science 349(6253), 1205-1208 (2015)

82. S. Nimmrichter, K. Hornberger, Stochastic extensions of the regularized Schrödinger-Newton equation. Phys. Rev. D 91(2), 024016 (2015)

83. S. Bera, S. Donadi, K. Lochan, T.P. Singh, A comparison between models of gravity induced decoherence. Found. Phys. 45(12), 1537-1560 (2015)

84. G. Gasbarri, M. Torol, S. Donadi, A. Bassi, Gravity induced wave function collapse. Phys. Rev. D 96(10), 104013 (2017)

85. O. Romero-Isart, A.C. Pflanzer, F. Blaser, R. Kaltenbaek, N. Kiesel, M. Aspelmeyer, J.I. Cirac, Large quantum superpositions and interference of massive nanometer-sized objects. Phys. Rev. Lett. 107(2), 020405 (2011)

86. L. Diosi, A universal master equation for the gravitational violation of quantum mechanics. Phys. Lett. A 120(8), 377-381 (1987)

87. R. Penrose, On gravity's role in quantum state reduction. Gen. Relativ. Gravit. 28(5), 581-600 (1996)

88. S. Bose, K. Jacobs, P.L. Knight, Preparation of nonclassical states in cavities with a moving mirror. Phys. Rev. A 56(5), 4175 (1997)

89. S. Bose, K. Jacobs, P.L. Knight, Scheme to probe the decoherence of a macroscopic object. Phys. Rev. A 59(5), 3204 (1999)

90. M.R. Vanner, I. Pikovski, G.D. Cole, M.S. Kim, Č. Brukner, K. Hammerer, G.J. Milburn, M. Aspelmeyer, Pulsed quantum optomechanics. Proc. National Acad. Sci. 108(39), 1618216187 (2011)

91. W. Marshall, C. Simon, R. Penrose, D. Bouwmeester, Towards quantum superpositions of a mirror. Phys. Rev. Lett. 91(13), 130401 (2003)

92. K. Hornberger, S. Uttenthaler, B. Brezger, L. Hackermüller, M. Arndt, A. Zeilinger, Collisional decoherence observed in matter wave interferometry. Phys. Rev. Lett. 90(16), 160401 (2003)

93. S.L. Adler, Gravitation and the noise needed in objective reduction models Quantum Nonlocality and Reality: 50 Years of Bell's Theorem ed M Bell and S Gao (2016) 
94. M. Bronstein, Republication of: Quantum theory of weak gravitational fields. Gen. Relativ. Gravit. 44(1), 267-283 (2012)

95. B.S. DeWitt, D. Bryce Seligman, The Global Approach to Quantum Field Theory (Vol. 114) (Oxford University Press, USA, 2003)

96. A. Peres, D.R. Terno, Hybrid classical-quantum dynamics. Phys. Rev. A 63(2), 022101 (2001)

97. C. Marletto, V. Vedral, Why we need to quantise everything, including gravity. npj Quantum Inf. 3(1), 1-5 (2017)

98. M. Carlesso, A. Bassi, M. Paternostro, H. Ulbricht, Testing the gravitational field generated by a quantum superposition. New J. Phys. 21(9), 093052 (2019)

99. M. Carlesso, M. Paternostro, H. Ulbricht, A. Bassi, When Cavendish meets Feynman: A quantum torsion balance for testing the quantumness of gravity (2017). arXiv preprint arXiv: 1710.08695

100. S. Bose, A. Mazumdar, G.W. Morley, H. Ulbricht, M. Torol, M. Paternostro, A.A. Geraci, P.F. Barker, M.S. Kim, G. Milburn, Spin entanglement witness for quantum gravity. Phys. Rev. Lett. 119(24), 240401 (2017)

101. C. Marletto, V. Vedral, Gravitationally induced entanglement between two massive particles is sufficient evidence of quantum effects in gravity. Phys. Rev. Lett. 119(24), 240402 (2017)

102. H. Miao, D. Martynov, H. Yang, A. Datta, Quantum correlations of light mediated by gravity. Phys. Rev. A 101(6), 063804 (2020)

103. W. Gerlach, O. Stern, Der experimentelle Nachweis des magnetischen Moments des Silberatoms. ZPhy 8(1), 110-111 (1922)

104. M. Utz, M.H. Levitt, N. Cooper, H. Ulbricht, Visualisation of quantum evolution in the SternGerlach and Rabi experiments. Phys. Chem. Chem. Phys. 17(5), 3867-3872 (2015)

105. B. Friedrich, D. Herschbach, Stern and Gerlach: How a bad cigar helped reorient atomic physics. Phys. Today 56(12), 53-59 (2003)

106. S. Machluf, Y. Japha, R. Folman, Coherent Stern-Gerlach momentum splitting on an atom chip. Nat. Commun. 4(1), 1-9 (2013)

Open Access This chapter is licensed under the terms of the Creative Commons Attribution 4.0 International License (http://creativecommons.org/licenses/by/4.0/), which permits use, sharing, adaptation, distribution and reproduction in any medium or format, as long as you give appropriate credit to the original author(s) and the source, provide a link to the Creative Commons license and indicate if changes were made.

The images or other third party material in this chapter are included in the chapter's Creative Commons license, unless indicated otherwise in a credit line to the material. If material is not included in the chapter's Creative Commons license and your intended use is not permitted by statutory regulation or exceeds the permitted use, you will need to obtain permission directly from the copyright holder. 